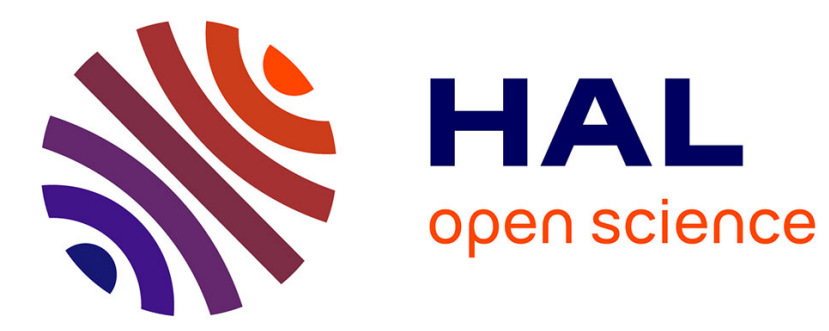

\title{
Glioplasticity in irritable bowel syndrome
}

\author{
N. L Lilli, L. Quénéhervé, S. Haddara, C. Brochard, P. Aubert, Malvyne \\ Rolli-Derkinderen, T Durand, Philippe Naveilhan, Jean-Benoit Hardouin, R. \\ de Giorgio, et al.
}

\section{- To cite this version:}

N. L Lilli, L. Quénéhervé, S. Haddara, C. Brochard, P. Aubert, et al.. Glioplasticity in irritable bowel syndrome. Neurogastroenterology \& Motility, 2018, 30 (4), pp.e13232. 10.1111/nmo.13232 . hal-03153591

\section{HAL Id: hal-03153591 \\ https://cnrs.hal.science/hal-03153591}

Submitted on 9 Mar 2021

HAL is a multi-disciplinary open access archive for the deposit and dissemination of scientific research documents, whether they are published or not. The documents may come from teaching and research institutions in France or abroad, or from public or private research centers.
L'archive ouverte pluridisciplinaire HAL, est destinée au dépôt et à la diffusion de documents scientifiques de niveau recherche, publiés ou non, émanant des établissements d'enseignement et de recherche français ou étrangers, des laboratoires publics ou privés. 


\title{
Glioplasticity in irritable bowel syndrome
}

\author{
N. L. Lilli ${ }^{1,2^{*}}$, L. Quénéhervé ${ }^{1,2^{*}}$, S. Haddara ${ }^{1,2}$, C. Brochard ${ }^{1}$, P. Aubert ${ }^{1,2}$ \\ M. Rolli-Derkinderen ${ }^{1,2}$, T. Durand ${ }^{1,2}$, P. Naveilhan ${ }^{1,2}$, J-B Hardouin ${ }^{3}$, R. De \\ Giorgio $^{4}$, G. Barbara4, S. Bruley des Varannes ${ }^{1,2}$, E. Coron ${ }^{1,2}$, M. Neunlist ${ }^{1,2^{*}}$
}

1Université de Nantes, INSERM, IMAD, The enteric nervous system in gut and brain disorders, Université Bretagne Loire, Nantes, France

2 Institut des Maladies de l'Appareil Digestif, IMAD, CHU Nantes, Hopital Hôtel-Dieu, Nantes, France

3Université de Nantes, INSERM, SPHERE, Université Bretagne Loire, Nantes, France

4Department of Medical and Surgical Sciences, St. Orsola-Malpighi

Hospital, University of Bologna, Bologna, Italy

*Contributed equally to this work.

\section{Correspondence}

Dr. Michel Neunlist, UMR Inserm U1235, University of Nantes, The enteric nervous system in gut and brain disorders, Institut des Maladies de l'Appareil Digestif, School of Medicine University of Nantes, Nantes, France.

Email: michel.neunlist@univ-nantes.fr

\section{Funding information}

Part of study was funded by an Almirall grant 'Defining IBS-C as a gliopathy' and a grant from Region Pays de la Loire (Vacarme).

Abbreviations: 7-AAD, 7-aminoactinomycin D; ATP, adenosine triphosphate; DMEM, Dulbecco's modified Eagle medium; EGC, enteric glial cells; ENS, enteric nervous system; HC, healthy control; $\mathrm{H} 1 \mathrm{R}$, histamine $\mathrm{H} 1$ receptor; IBS, irritable bowel syndrome; IBS-C, constipation-predominant IBS; IBS-D, diarrhea-predominant IBS; IBS-M, mixed-IBS; qPCR, quantitative PCR. 


\begin{abstract}
Background: Growing evidence indicates a wide array of cellular remodeling in the mucosal microenvironment during irritable bowel syndrome (IBS), which possibly con- tributes to pathophysiology and symptom generation. Here, we investigated whether enteric glial cells (EGC) may be altered, and which factors/mechanisms lead to these changes.

Methods: Colonic mucosal biopsies of IBS patients (13 IBS-Constipation [IBS-C]; 10 IBSDiarrhea [IBS-D]; 11 IBS-Mixed [IBS-M]) and 24 healthy controls (HC) were ana- lyzed. Expression of $S 100 \beta$ and GFAP was measured. Cultured rat EGC were incubated with supernatants from mucosal biopsies, then proliferation and $\mathrm{Ca} 2+$ response to ATP were analyzed using flow cytometry and $\mathrm{Ca} 2+$ imaging. Histamine and histamine 1-receptor $(\mathrm{H} 1 \mathrm{R})$ involvement in the effects of supernatant upon EGC was analyzed.

Key Results: Compared to HC, the mucosal area immunoreactive for $\mathrm{S} 100 \beta$ was significantly reduced in biopsies of IBS patients, independently of the IBS subtype. IBS-C supernatants reduced EGC proliferation and IBS-D and IBS-M supernatants reduced $\mathrm{Ca} 2+$ response to ATP in EGC. EGC expressed H1R and the effects of supernatant upon Ca2+ response to ATP in EGC were blocked by pyrilamine and reproduced by histamine via H1R. IBS supernatants reduced mRNA expression of connexin-43. The S100 $\beta$-stained area was negatively correlated with the frequency and intensity of pain and bloating.

Conclusion and Inferences: Changes in EGC occur in IBS, involving mucosal soluble factors. Histamine, via activation of H1R-dependent pathways, partly mediates altered $\mathrm{Ca} 2+$ response to ATP in EGC. These changes may contribute to the pathophysiology and the perception of pain and bloating in patients with IBS.
\end{abstract}

\title{
KEYWORDS
}

Ca2+ response, enteric glial cells, histamine, irritable bowel syndrome 


\section{1| INTRODUCTION}

Irritable bowel syndrome (IBS) is a functional intestinal disease characterized by chronic abdominal pain and altered bowel habits with a world prevalence of about $10 \%-25 \%$ worldwide.1 Subtypes of IBS have been defined according to bowel habit patterns, including IBS with predominant constipation (IBS-C), predominant diar- rhea (IBS-D), mixed bowel habits (IBS-M), and 'unclassified' (IBS-U) in patients who do not meet diagnostic criteria for IBS-C, D, or M.2 Although the etiology of IBS remains unknown, major advances have been achieved over the past few years enabling a better pathophysiological understanding. In particular, a remodeling of the gut mucosal cellular microenvironment, such as increased mast cells and $T$ cell activation3 along with histamine, protease, and cytokine release in the colonic mucosa, 3,4 has been reported in IBS. In addi- tion, some IBS subsets of patients are characterized by increased paracellular permeability, 5 altered enteroendocrine cell density, 6 and enteric nervous system (ENS) neuroplasticity. Recent studies have described increased neuronal fiber outgrowth, 7 density of sub- stance $P, 8$ or vanilloid receptor 1 (TRPV1) 9 nerve fibers. The impact of mucosal factors on the ENS is supported by the evidence that nerve exposure to soluble mediators in supernatant obtained from mucosal biopsies of IBS patients increases the excitability of enteric neurons10 and evokes neuroplastic changes in the ENS through the contribution of mucosal histamine, serotonin, protease, or nerve growth factor.11

Besides enteric neurons, the ENS is composed of enteric glial cells (EGC) that outnumber them by a factor of 1.3 to 1.9 and 5.9 to 7.0 in the human submucosal plexus and myenteric plexus, respec- tively.12 Enteric glial cells are central regulators of neurons, intesti- nal epithelial barrier, and gut functions, such as motility,13,14 which have been described as altered in an experimental model remi- niscent of IBS.15 However, to the best of our knowledge, changes in EGC in patients with IBS have never been thoroughly studied previously.

Enteric glial cells regulate neuronal processes such as neuronal survival16 and neuromediator expression.12,17-20 Conversely, en- teric neurons can also communicate to EGC, in particular, via ad- enosine triphosphate (ATP)-dependent P2Y4/P2Y1 pathways.21,22 Activation of EGC by such pathways leads to glio-glio communi- cation via connexin-43dependent signaling.23 Interestingly, glial- specific ablation of connexin-43 leads to a reduced ATP-induced glio-glio Ca2+-dependent communication and ultimately to a re-duced intestinal motility.24 Consistently, loss of EGC has also been reported to be associated with intestinal dysmotility in both human and animal models of glia ablation.25-27 In addition to neu- ronal/motor functions, EGC also control intestinal barrier func- tions, such as paracellular permeability,17,28,29 which can be altered in IBS.5

This study was designed to characterize putative EGC glio- plasticity in the colonic mucosa of IBS patients and identify the underlying mediators, focusing on those produced by the mucosal microenvironment. Specifically, we aimed to (i) characterize EGC phenotype in a bio-collection of colonic biopsies from IBS patients and healthy controls ( $\mathrm{HC}$ ); (ii) determine whether IBS supernatants could induce EGC phenotypic and functional alterations; and (iii) identify soluble factor(s) and cellular targets responsible for these changes. 


\section{2 | MATERIALS AND METHODS}

\section{1 | Patients and biopsies}

Patients referred for colonoscopy for digestive symptoms sugges- tive of IBS were enrolled in the study. After giving their informed consent according to the guidelines of the French Ethics Committee for Research on Humans (DC-2008-402), they completed a Rome III questionnaire 30 and a bowel habit assessment in order to diagnose IBS according to Rome criteria, and to determine the IBS subtype. The inclusion criteria included discomfort or abdominal pain at least 3 days per month during the last 3 months, discomfort or pain for at least 6 months, associated with 2 or more of the following: improve- ment of symptoms with defecation, onset associated with a change in frequency of stool and onset associated with a change in consist- ency of stool. If the patient had hard stools or separate lumpy stools form over $25 \%$ of the time, he/she was labeled as IBS-C; if the stool was loose or watery over $25 \%$ of the time, he/she was labeled as IBS-D; and if these 2 types of stools were present over $25 \%$ of the time, he/she was included in the IBS-M subgroup. The severity and frequency of abdominal pain and bloating were assessed on a scale from 0 to 4 , adapted from previous studies on IBS.5 HC answered the same questionnaire and had no digestive complaints. Clinical data were stored in an electronic database (Integralis). Biopsies ( $n=9 /$ patient) were obtained for this study using standard biopsy forceps without needle (FB230U; Olympus co., Rungis, France) in the left colon, between 30 and $40 \mathrm{~cm}$ from anal verge during the colonoscopy. The biopsies were then placed into physiological serum on ice and immediately transferred to the laboratory.

\subsection{Microdissection and immunohistochemistry}

Microdissection was performed as previously described31 in 3 biop- sies. Each whole-mount preparation was fixed in paraformaldehyde $4 \%$ for 3 hours, washed 3 times with PBS (phosphate-buffered saline) $1 \times$ and then stored at $4^{\circ} \mathrm{C}$ in PBS/NaN3 for later immunohistochem- istry. Specimens of mucosa and submucosa were permeabilized for 3 hours in PBS/NaN3 containing 1\% (v/v) Triton X-100 and 10\% (v/v) horse serum and then incubated overnight with primary antibodies diluted in permeabilization solution. Wholemount preparations were then rinsed 3 times with PBS for 30 minutes and secondary antibodies diluted in PBS/NaN3 containing 10\% (v/v) horse serum were added for 3 hours. Primary and secondary antibodies used in all experiments are listed in supporting information (Table S1). Whole specimens of mucosa and submucosa were viewed under an Axio Zoom.V16 ster- eomicroscope (Zeiss, Marly Le Roi, France). The area occupied by EGC in colonic mucosa from IBS patients and $\mathrm{HC}$ was quantified as the $\mathrm{S} 100 \beta$ immunoreactive area in one field of observation $(0,33 \mathrm{~cm} 2)$ using ImageJ software. The results are expressed as the mean of 3 fields observed for biopsy.

\section{3 | Western blot analysis}

Two biopsies per patient were recovered in RA1 lysis buffer to sepa- rate RNA and proteins. The same process was used for cultures of EGC treated either with supernatants from biopsies or with drugs.

Samples were processed for electrophoresis using the SDS-PAGE buffer kit (Invitrogen, SaintAubin, France) and separated on 4\%-12\% BisTris gel (Invitrogen). Proteins were transferred to nitrocellulose mem- branes with the iBlot system (Life Technologies, Carlsbad, CA, USA). After being blocked with Tris-buffered saline, $0.1 \%$ Tween 20 and $5 \%$ non-fat dry milk for 1 hour, blots were incubated overnight at $4^{\circ} \mathrm{C}$ with primary antibodies diluted in Tris-buffered saline and $5 \%$ non-fat dry milk for rabbit anti-GFAP (glial fibrillary acidic protein) (Dako, Les 
Ulis, France, 1/2000) and rabbit anti-S100 $\beta$ (Dako, 1/500). Immunoblots were probed with the appropriate horseradish peroxidase conjugated secondary antibodies (Life Technologies) and visualized by chemilumi- nescence (Clarity Western ECL Substrate; Bio-Rad, Hercules, CA, USA) using a Gel-Doc imager and the Image Lab Software (Bio-Rad, Hercules, CA, USA). The value of total protein immunoreactivity was normalized to the glyceraldehyde-3phosphate dehydrogenase (GAPDH) immuno- reactivity and expressed as a percentage of the average of controls.

\subsection{Quantitative polymerase chain reaction (PCR)}

RNA from biopsies or cultures of EGC treated with supernatants from biopsies or with drugs was isolated using the Nucleo Spin RNA Triprep Kit or Clean up (Macherey-Nagel, Düren, Germany), respectively, according to the manufacturer's instructions. Potential genomic DNA contamination was removed by treatment with TurboTM DNase (Ambion Inc., Austin, TX, USA) and RNA was quantified using an ND-1000 UV-Vis spectrophotometer (Nanodrop Technologies, Wilmington, DE, USA). cDNA was synthesized from $1 \mu \mathrm{g}$ total RNA using the Super Script III Reverse Transcriptase System kit (Invitrogen) and diluted to a final concentration of $8 \mathrm{ng}$ eq RNA/ $\mu$ L. qPCR was performed using StepOne Plus (Life Technologies) detection system with Fast SYBR Green (Life Technologies) master mix. The PCR signal was normalized against $\mathrm{S} 6$ as reference gene to control for variabil- ity in the amount and quality of the RNA. The sense and antisense oligonucleotide primers used in this study are shown in supporting information Table S1.

\section{5 | Biopsies supernatant production}

Four biopsies were rapidly weighted, and transferred in plastic tubes that were completed with Krebs Hepes solution ( $1 \mathrm{~mL}$ of Krebs Hepes for $30 \mathrm{mg}$ of biopsy). After 20-minute incubation at $37^{\circ} \mathrm{C}$ and being continuously oxygenated (95\% O2/5\% CO2), the solution was re- moved and centrifuged at 11000 RCF (relative centrifugal force) for 1 minute before being filtered with centrifuge tube filters ( $0.22 \mathrm{~mm}$, SPIN-X; Corning, New York, USA). Aliquots of $300 \mu \mathrm{L}$ of supernatant were stored at $80^{\circ} \mathrm{C}$ until assays.

\section{6 | Cell cultures}

Embryonic EGC of rat (cell-lineage JUG2 Inserm U1235) were iso- lated from ENS primary culture derived from rat embryonic in- testine (E15)32 according to a procedure previously described.33 Briefly, after 13 days of culture, primary cultures were trypsinized and seeded in serum-containing media after differential centrifuga- tion. Following 7 days of culture, isolated areas of morphological glial cells-like were trypsinized using cloning cylinder and seeded in culture flask in serum-containing media. They were immunoreactive for GFAP, Sox10, and S-100 $\beta$, all glial markers, but not for Tuj-III, PGP9.5, neuronal markers, and smooth muscle actin, a myofibro- blast marker.

Enteric glial cells were cultured in $25 \mathrm{~cm} 2$ flask in Dulbecco's modified Eagle medium (DMEM) Glutamax medium (4.5 g/L glucose; Invitrogen) or DMEM medium (4.5 g/L glucose; Invitrogen) supple- mented with $10 \%$ heat-inactivated fetal calf serum (FCS) (Abcys, Paris, France), $2 \mathrm{mmol} \mathrm{L}-1$ glutamine (Invitrogen) and $50 \mathrm{IU} / \mathrm{mL}$ penicillin, and $50 \mathrm{mg} / \mathrm{mL}$ streptomycin (Invitrogen). For Western blot analysis, EGC were seeded at a density of 30000 cells/well in $100 \mu \mathrm{L}$ of medium on a 96-well plate, and cultured for 1 day prior in- cubation with supernatants, drugs, or control medium (added daily) for 2 days.

\section{7 | Flow cytometry analysis}

Enteric glial cells were seeded at a density of 10000 cells/well in $200 \mu \mathrm{L}$ of medium on a 96well plate. Addition of $20 \mu \mathrm{L}$ of IBS or HC supernatant was performed at day 1,3 , and 5 . At day 7 , cell culture supernatants were recovered and transferred in a 96-well conical bottom 
plate and then cen- trifuged for 2 minutes at $2000 \mathrm{~g}$. Enteric glial cells were washed with PBS $1 \mathrm{X}$ and trypsinized with $0.25 \%$ of trypsin EDTA (GIBCO ThermoFisher Scientific, Cillebon sur Yvette, France) for 5 minutes at $37^{\circ} \mathrm{C}$. The trypsin reaction was stopped by adding medium containing $10 \%$ FCS and then EGC were recovered and placed in the 96 -well conical bottom plate. The 96-well conical bottom plate was centrifuged for 2 minutes at $2000 \mathrm{~g}$ and then PBS/EDTA was added after supernatant removal. Enteric glial cells were re-suspended with $10 \mu \mathrm{g} / \mathrm{mL}$ of 7-aminoactinomycin D (7-AAD) (Sigma) and then fluorescent labeling was measured using a FACS LSR II (BD Biosciences, Le Pont de Claix, France) and analyzed with BD FACS Diva software.

\section{8 | Calcium flux assays}

Enteric glial cells were seeded at a density of 4000 cells/well in $200 \mu \mathrm{L}$ of medium on a 96well plate. The incubation of EGC culture with $20 \mu \mathrm{L}$ of supernatants or drug/antagonists was performed at day 1 and day 2. After 48 hours of incubation, EGC were washed with PBS and incubated at $37^{\circ} \mathrm{C}$ in fresh warmed Hanks' balanced salt solution (HBSS) contain- ing 0.5 umol L-1 fluo-4 AM (wavelength of absorption: $494 \mathrm{~nm}$; wave- length of emission: $516 \mathrm{~nm}$ ) for 60 minutes. After washing with HBSS, the 96-well plate was placed under Olympus IX 50 inverted microscope with a Olympus DP 50 digital imaging system and Ca2+ flux analysis was performed one well at the time after addition of ATP (final concentra- tion of $2 \mathrm{mmol} L-1$ ). For each well, a sufficiently dense field of cells was selected to analyze Ca2+ flux of 30 cells. The acquisition was fixed with an exposure time of 1 second. The experiment was then recorded for 120 seconds ( 25 images/seconds) using Cell B software.

\section{9 | Calcium imaging analysis}

Movies were converted in a sequence of images (JPEG format) with VirtualDub software and analyzed using the ImageJ software with which the outline of 30 individualized cells was traced. Change in fluo- rescence $(F)$ (calculated as $[\triangle F / F=[F M A X-F M I N] / F M A X)$ was calculated. All values were expressed as the percentage of the average of control wells for each separate experiment.

\subsection{0 | Immunohistochemical studies}

Enteric glial cells were seeded at a density of 4000 cells/well in $200 \mu \mathrm{L}$ of medium on a 96well plate and cultured during 48 hours. Enteric glial cells were subsequently fixed in paraformaldehyde $4 \%$ for 10 minutes, washed 3 times with PBS, incubated 30 minutes with PBS/NaN3 containing 10\% (v/v) horse serum, and subsequently, 1 hour with primary antibodies. Enteric glial cells were rinsed 3 times with PBS for 5 minutes and then secondary antibodies diluted in PBS/NaN3 containing 10\% (v/v) horse serum were added for 30 minutes. Nuclei were stained with 4-6-diamidino-2-phenylindole (Dapi) (1:5000, Sigma). Primary and secondary antibodies used in all experiments are listed in Table S2.

Fluorescently labeled EGC were viewed under an Axio Zoom.V16 stereomicroscope (Zeiss, Marly Le Roi, France).

\subsection{1 | Pharmacological studies}

All drugs were diluted and reconstituted in culture medium. PAR-2 agonist (SLIGRL; Biochem) was used at final concentrations of 10-1, 10-3, 10-5, and 10-7 $\mu \mathrm{mol} L-1$. Histamine (Sigma) and serotonin (Sigma) were used at final concentrations of $10-2,1$, and $100 \mu \mathrm{mol} L-1$. H-1 receptor antagonist pyrilamine (Sigma) was used at final concentration of $1 \mu \mathrm{mol} L-1$.

\subsection{2 | Statistical analysis}

All graphs and statistical significance evaluations were performed using GraphPad Prism Software (GraphPad Software, Inc., La Jolla, CA, USA). A Fisher's exact test was used to analyze clinical variables expressed in percentage. Differences between groups were calcu- 
lated by a 2-tailed Student's $t$ test for non-parametric and unpaired data or Mann-Whitney U test and Kruskal-Wallis non-parametric ANOVA test followed by Dunn's posttest. For the H1R antagonist ef- fect experiments, a 2-way ANOVA test followed by a Bonferroni post hoc test was used. The relation between quantitative variables and clinical data was calculated using a Pearson's correlation coefficient test. Values of $P \leq .05$ were considered statistically significant.

\section{3 | RESULTS}

\section{1 | Study patients}

From December 2013 to March 2015, 34 IBS patients and 24 HC were included. In the overall population, mean age was $48.6 \pm 14.8$ years and male:female distribution was $36: 58$ (62.1\% female). The clinical characteristics of HC and IBS patients are re-ported in Table 1. There was no difference in demographic factors and in intensity and frequency of abdominal pain and bloating be- tween IBS subtypes.

TABLE 1 Characteristics of the study population

\begin{tabular}{|c|c|c|c|c|c|}
\hline & $\begin{array}{l}\text { Healthy controls } \\
(n=24) n(\%) \text { or mean (SD) }\end{array}$ & $\begin{array}{l}\text { All IBS }(n=34) \\
n(\%) \text { or mean (SD) }\end{array}$ & $\begin{array}{l}\text { IBS-C }(n=13) \\
n(\%) \text { or mean }(S D)\end{array}$ & $\begin{array}{l}\text { IBS-D }(n=10) \\
n(\%) \text { or mean (SD) }\end{array}$ & $\begin{array}{l}\text { IBS-M }(n=11) \\
n(\%) \text { or mean (SD) }\end{array}$ \\
\hline Age (y) & $45.2(13.3)$ & $51.0(15.6)$ & $46.8(13.6)$ & $55.1(14.3)$ & $52.2(18.8)$ \\
\hline Sex male & $13(54.0)$ & $9(26.5)^{*}$ & $3(23.1)$ & $2(20.0)$ & $4(36.4)$ \\
\hline Depression & $4(17.4)$ & $12(40.0)$ & $5(38.4)$ & $3(33.3)$ & $5(55.6)$ \\
\hline Pain intensity & $0.08(0.4)$ & $1.81(0.8)^{*}$ & $1.6(0.8)$ & $2.1(0.7)$ & $1.8(0.9)$ \\
\hline Bloating intensity & $0.42(0.6)$ & $1.54(0.8)^{*}$ & $1.6(0.9)$ & $1.5(0.9)$ & $1.5(0.9)$ \\
\hline Pain frequency & $0.13(0.6)$ & $2.3(1.1)^{*}$ & $2.3(0.9)$ & $2.5(1.0)$ & $2.2(1.3)$ \\
\hline Bloating frequency & $0.75(1.3)$ & $2.5(1.3)^{*}$ & $2.5(1.2)$ & $2.7(1.4)$ & $2.3(1.4)$ \\
\hline
\end{tabular}

$* P<.05$ compared to healthy controls.

(A)

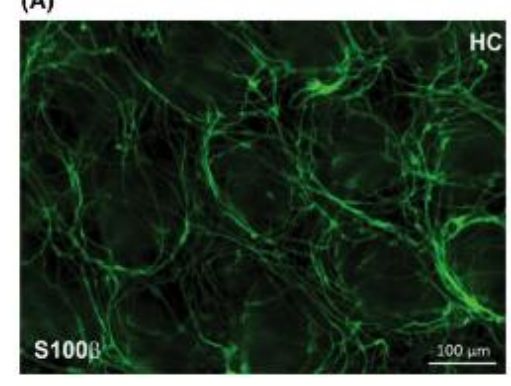

(B)

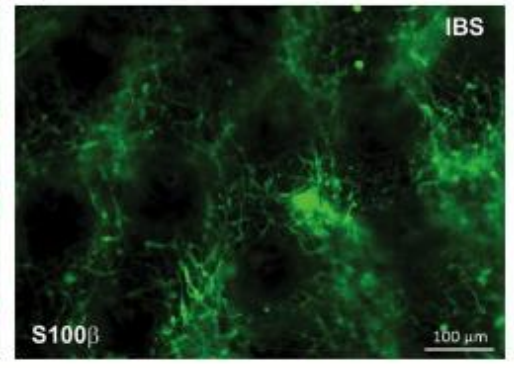

(C)

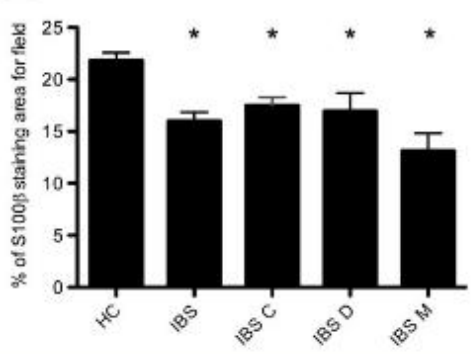

FIGURE 1 Characterization of enteric glial network in colonic biopsies from healthy control and IBS patients. Representative images of

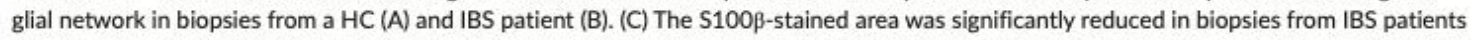
compared to HC, whatever the IBS subtype (mean of 3 fields for biopsy observed). Data are expressed as mean values $\pm S E M$ of $n=15 \mathrm{HC}$ and $n=26$ IBS patients $(n=10 \text { IBS-C; } n=8 \text { IBS-D; } n=8 \text { IBS-M })^{*} P<.05$

\section{2 | Mucosal enteric glial network}

Changes in $\mathrm{S} 100 \beta$ protein expression were investigated in the mu-cosa from biopsies of $\mathrm{HC}$ and IBS patients using immunohistochemical methods (Figure 1A-B). Quantitative analysis showed that the $S 100 \beta$ staining area was significantly reduced by $27 \%$ in biopsies from IBS patients as compared to $\mathrm{HC}(P<.0001)$ (Figure $1 C)$. A similar reduc- tion was shown in all subtypes of IBS. To verify that the difference observed depended on the change of glial intensity and not on the modification of the mucosal architecture, the number of crypts per field of observation was counted and no difference between IBS patients and $\mathrm{HC}$ was detected (data not shown). Significant negative correlations were found between the $\mathrm{S} 100 \beta$ - 
stained area and the fre- quency $(r=-.42 ; P<.05)$ and intensity $(r=-.48 ; P<.05)$ of pain and bloating $(r=-.37 ; P<.05)$ in all IBS subtypes.

There was no difference in S100 $\beta$ and GFAP expression in all IBS subtypes as assessed by Western blot analysis (data not shown) and PCR. However, S100 $\beta$ mRNA tended to be linearly correlated with $S 100 \beta$ mucosa density (Figure S1A). Furthermore, S100 $\beta$ was also moderately correlated with connexin-43 and TNF $\alpha$ expression (Figure S1B and S1C).

\section{3 | Effect of mucosal supernatants on EGC proliferation and phenotype}

To determine whether soluble factors produced by biopsies were able to induce changes in EGC function and phenotype, we first investi- gated the effect of IBS supernatants on EGC proliferation.

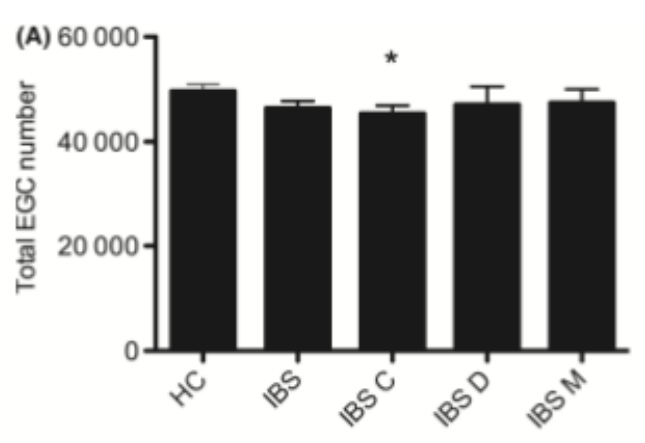

(B)

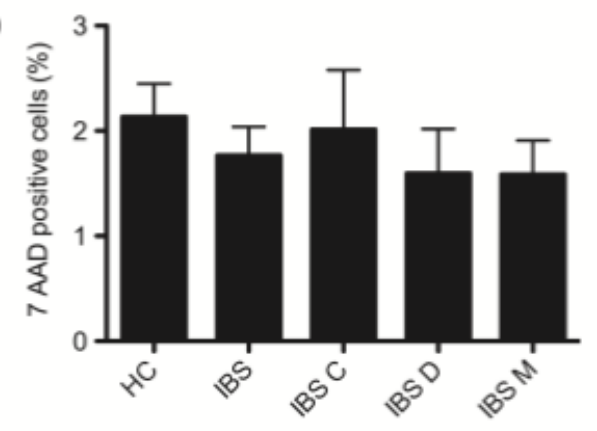

(C)

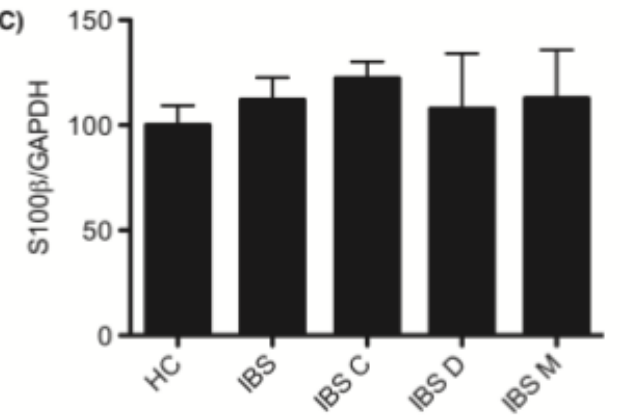

FIGURE 2 Effect of biopsies supernatant on EGC functions and phenotype. (A) There was no difference between the number of EGC treated for 6 days with IBS supernatants and EGC treated with $\mathrm{HC}$ supernatants. EGC number was significantly reduced in case of treatment with IBS-C supernatants as compared with EGC treated with $\mathrm{HC}$ supernatants. Data are expressed as mean values $\pm \mathrm{SEM}$ of $n=23 \mathrm{HC}$ and $n=29$ IBS patients ( $n=12$ IBS-C; $n=8$ IBS-D; $\mathrm{n}=9$ IBS-M) ${ }^{*} P<.05$. (B) There was no difference in the proportion of 7-AAD-positive cells to total cells after treatment with IBS and $\mathrm{HC}$ supernatants. (C) There was no difference in $\mathrm{S} 100 \beta$ expression as measured by Western blot in EGC cultured with IBS and HC supernatants. Data are expressed as mean values $\pm S E M$, normalized to $\mathrm{HC}$, of $n=9 \mathrm{HC}$ and $n=19$ IBS patients ( $n=5$ IBS-C; $n=7$ IBS-D; $n=7$ IBS-M)
Following treatment of EGC cultures with IBS and HC superna- tants, EGC cell number was evaluated (Figure 2A). The number of EGC was significantly reduced by $9 \%$ in cultures treated with IBS-C super- natants, but not IBS-M or IBS-D supernatants, as compared to HC. In addition, the reduction of EGC number induced by IBS-C super- natants was not associated with an increase in cell death (Figure 2B) as assessed by measuring the proportion of 7-AAD-positive EGC. There was a modest negative correlation between the number of EGC cultured with IBS and HC supernatants and the frequency of pain $(r=-.27 ; P<.05)$. Treatment of EGC with IBS supernatants did not change the ex-pression of $S 100 \beta$ (Figure 2C) or GFAP (data not shown) proteins as measured by Western blot.

\section{4 | Effects of mucosal supernatants on ATP- induced $\mathrm{Ca} 2+$ response of EGC} We next aimed to determine whether incubation of EGC ( 48 hours) with IBS supernatants could modify their $\mathrm{Ca} 2+$ response to ATP. The amplitude of $\mathrm{Ca} 2+$ response to ATP in EGC cultured with IBS superna- tants was significantly decreased as compared to the response in $\mathrm{EGC}$ cultured with $\mathrm{HC}$ supernatants (Figure 3A-B). These changes in $\mathrm{Ca} 2+$ response to ATP were dependent on IBS subtype. Indeed, the mean amplitude of $\mathrm{Ca} 2+$ flux was significantly reduced in EGC cultured with IBS-D and IBS-M supernatants, but not IBS-C, as compared 

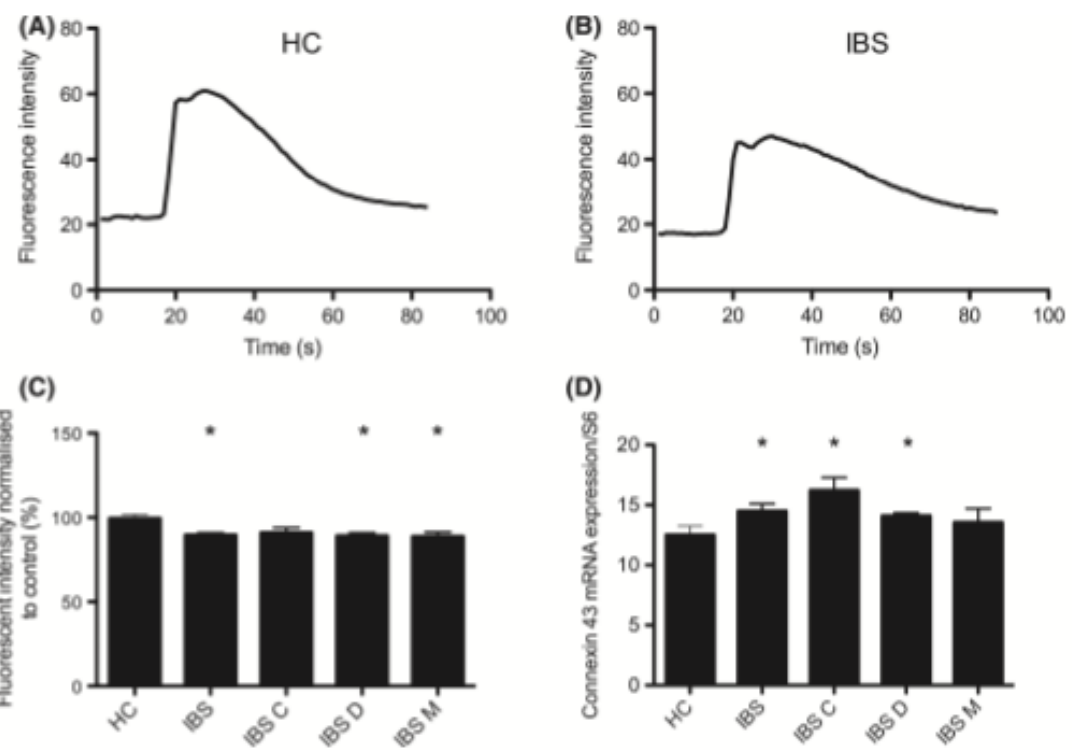

\begin{abstract}
FIGURE 3 Intracellular $\mathrm{Ca}^{2+}$ response to ATP in EGC cultured with IBS and $\mathrm{HC}$ supernatants. (A, B) Graphics representatives of $\mathrm{Ca}^{2+}$ response to ATP in one enteric glial cell after treatment (48 hours) with HC and IBS supernatants. (C) The amplitude of fluo-4 fluorescence intensity in EGC cultured with IBS supernatants, particularly with IBS-D and IBS-M supernatants, was significantly decreased compared to EGC cultured with HC supernatants. Data are expressed as mean values \pm SEM normalized to $\mathrm{HC}$, of $n=11 \mathrm{HC}$ and $n=20$ IBS $(n=6$ IBS-C; $n=7$ IBS-D; $\mathbf{n}=7$ IBS-M). ${ }^{*} P \leq .05$ (D) mRNA expression of connexin- 43 was significantly increased in EGC cultured with IBS supernatants (48 hours) compared to EGC cultured with HC supernatants, especially in EGC cultured with IBS-C and IBS-D supernatants. Data are expressed as mean values \pm SEM of $n=7$ HC and $n=17$ IBS ( $n=5$ IBS-C; $n=5$ IBS-D; $n=7$ IBS-M). $*$ $\leq .05$
\end{abstract}

to $\mathrm{HC}$ (Figure $3 \mathrm{C}$ ). In addition, the resting Ca2+ level was not different in EGC treated with IBS or HC supernatants (data not shown). Furthermore, acute exposure of EGC to IBS $(n=15)$ or $\mathrm{HC}(\mathrm{n}=11)$ supernatants did not induce changes in Ca2+ level (data not shown).

Interestingly, the amplitude of $\mathrm{Ca} 2+$ response to ATP was negatively correlated with pain intensity and frequency (respectively $r=-.53 ; P<.05$ and $r=-.40 ; P<.05$ ). As connexin-43 plays a role in $\mathrm{Ca} 2+$ re- sponse to ATP in EGC, we aimed at determining whether incubation of EGC with IBS and $\mathrm{HC}$ supernatants could modulate its expression. Connexin-43 mRNA expression was significantly increased by $16 \%$ in EGC cultured with IBS supernatants compared to HC. IBS subgroup analysis demonstrated that connexin-43 expression was significantly increased in EGC incubated with IBS-C and IBS-D supernatants as compared to HC (Figure 3D).

\title{
3.5 Mediators involved in supernatant effect upon Ca2+ response to ATP in EGC
} We next aimed to identify a candidate involved in supernatant effects upon $\mathrm{Ca} 2+$ response to ATP in EGC. Previous studies have identified mediators such as histamine, serotonin, and proteases as superna- tants mediators that could modulate ENS functions, and in particular neuronal excitability.10 Based on these findings, we tested the impact of EGC incubation with these mediators on Ca2+ response to ATP in EGC. Among the mediators tested, SLIGRL, serotonin, and histamine, only the latter was able to change Ca2+ response to ATP as compared to control. Indeed, 48-hour incubation of EGC with histamine $(1 \mu \mathrm{mol} L-1)$ reduced Ca2+ flux amplitude by $21 \%$ (Figure 4 ).

The expression of histamine receptor on EGC was established by immunohistochemical methods. We first assessed expression of H1R in submucosal plexus of HC and IBS biopsies. Using confocal micros- copy, $\mathrm{H} 1 \mathrm{R}$ co-localized with $\mathrm{S} 100 \beta$ immunoreactivity at the surface membrane of EGC (Figure 5). Using quantitative immunohistochemis- try, levels of H1Rimmunoreactivity were also identical in IBS and HC EGC (data not shown). 

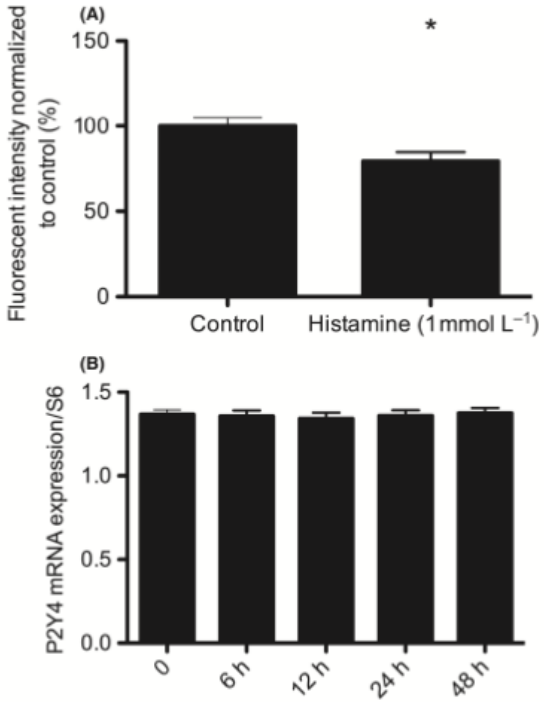

FIGURE 4 Identification of histamine as mediator involved in modification of $\mathrm{Ca}^{2+}$ response to ATP in EGC. (A) The amplitude of fluo-4 fluorescence intensity in EGC cultured (48 hours) with histamine $\left(1 \mu \mathrm{mol} \mathrm{L} \mathrm{L}^{-1}\right)$ was decreased compared to control. Data are expressed as mean values \pm SEM normalized to control, of $n=11$ independent experiments. ${ }^{*} P<.05$. (B) Time-course analysis of P2Y4 mRNA expression in EGC treated ( 48 hours, 24 hours, 12 hours, and 6 hours) with histamine $\left(1 \mu \mathrm{mol} \mathrm{L}^{-1}\right)$. No difference was observed at any time compared to control Data are expressed as mean values \pm SEM of $n=3$ independent experiments
Pharmacological approaches were applied to establish whether the effects of histamine and IBS supernatants upon $\mathrm{Ca} 2+$ response to ATP were mediated via H1R activation. Pretreatment of EGC with pyrila- mine (1 $\mu \mathrm{mol} L-1$ ) significantly reversed the changes in $\mathrm{Ca} 2+$ response to ATP caused by histamine. Moreover, pyrilamine did not modify Ca2+ response to ATP in EGC not treated with histamine (Figure 6A). Finally, the effects of IBS supernatants upon Ca2+ responses to ATP in EGC were prevented by pyrilamine (Figure 6B). To verify that the decrease in Ca2+ response to ATP in EGC cultured with histamine $(1 \mu \mathrm{mol} L-1)$ was not due to a modification in the expression of ATP receptors, we performed a time-course experiment to study the expression of mRNA of P2Y4 in EGC treated with histamine ( $1 \mu \mathrm{mol} \mathrm{L}-1)$ during 48 hours, 24 hours, 12 hours, and 6 hours. No difference was observed in the expression of mRNA of P2Y4 in EGC treated with histamine at any time compared to control (Figure 4B). Finally, histamine also did not modify connexin-43

expression and EGC proliferation (data not shown).
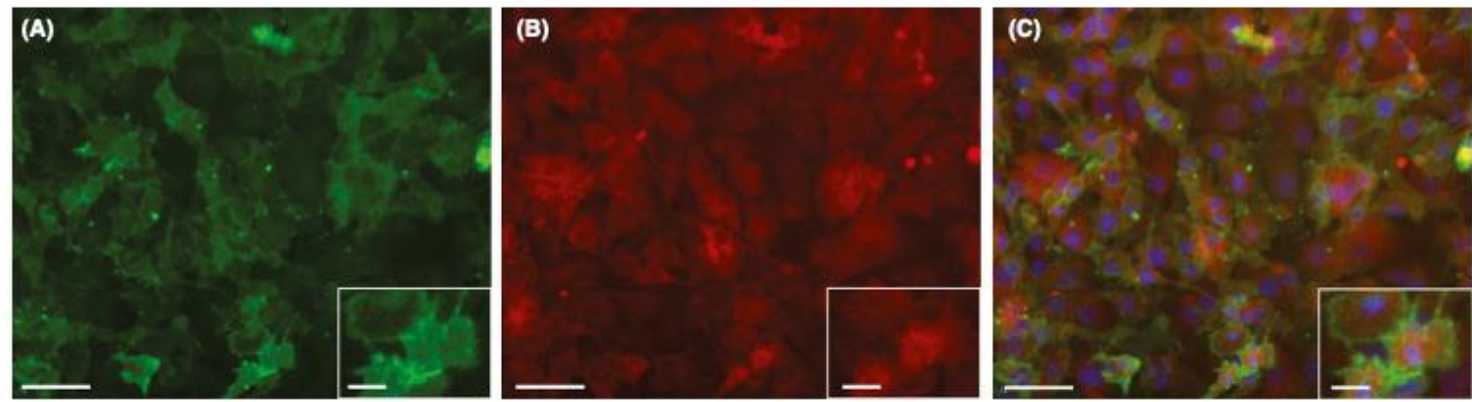

FIGURE 5 Characterization of H1R expression in enteric glial cells cultures. (A-C) Representative images of EGC expression of S100 $\beta$ (A; green) and $\mathrm{H} 1 \mathrm{R}(\mathrm{B}$; red) proteins. (C) Overlay of A-B images and 4-6-diamidino-2-phenylindole-labeled stained nuclei (blue). Scale bar $=50 \mu \mathrm{m}$. Enlarged view of boxed areas. Scale bar $=25 \mu \mathrm{m}$

(A)

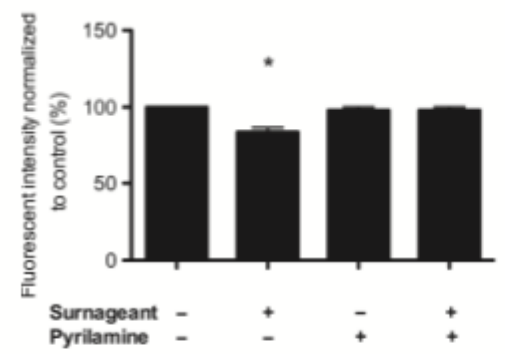

(B)

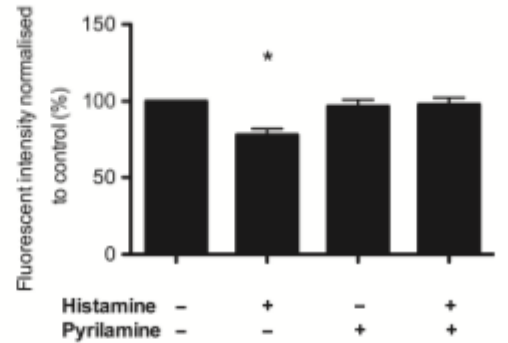

FIGURE 6 Effect of histamine or supernatant from IBS patients on $\mathrm{Ca}^{2+}$ response to ATP in EGC. (A) The pretreatment with pyrilamine $\left(1 \mu \mathrm{mol} \mathrm{L}{ }^{-1}\right)$ reversed the significant decrease of $\mathrm{Ca}^{2+}$ response to ATP in EGC treated (48 hours) with histamine $\left(1 \mu \mathrm{mol} \mathrm{L}{ }^{-1}\right)$ compared to control. Data are expressed as mean values \pm SEM normalized to control, of $n=6$ independent experiments. ${ }^{*} P<.05$. (B) The pretreatment with pyrilamine $\left(1 \mu \mathrm{mol} \mathrm{L}{ }^{-1}\right)$ reversed the significant decrease of $\mathrm{Ca}^{2+}$ response to ATP in EGC cultured (48 hours) with IBS supernatants. Data are expressed as mean values \pm SEM normalized to $\mathrm{HC}$, of $n=7$ independent experiments. ${ }^{*} P<.05$ 


\section{4 | DISCUSSION}

This study highlights the occurrence of enteric glioplasticity in the colonic mucosa of IBS patients and identifies histamine as a con- tributing factor underlying these changes. We first showed a re- duced mucosal labeling of $S 100 \beta$ in IBS biopsies as compared to HC, independent of IBS subtypes. Importantly, the S100 $\beta$-stained area was negatively correlated with the frequency and intensity of pain and bloating. Next, IBS-C supernatants, but not IBS$D$ and IBS-M, reduced EGC proliferation. Furthermore, IBS-D and IBS-M superna- tants, but not IBS-C, reduced $\mathrm{Ca} 2+$ response to ATP in EGC. We next showed that these latter changes were blocked by H1R antagonist and reproduced by histamine but not serotonin or PAR-2 agonist (SLIGRL). Overall, our study reveals the occurrence of changes in EGC in IBS that are dependent on the clinical phenotype thus sug- gesting that EGC abnormalities could contribute to IBS pathophysi- ology and symptoms.

An important finding of our study was the identification of changes in EGC protein expression in the mucosa of IBS patients. In particular, we showed that $S 100 \beta$ staining was significantly reduced in IBS as compared to HC, although protein and mRNA expression of $S 100 \beta$ remained unaltered. This discrepancy could result from the different methods used. Indeed, immunohistochemistry specifically evaluated EGC (identified by their S100ß immunoreactivity), whereas PCR and Western blot techniques take into account other cell types contained in biopsies besides EGC (immune cells, epithelial cells, and endothe- lial cells) during normalization. In addition, biopsies also differed one from another in terms of cellular distribution according to their size and depth. To the best of our knowledge, only a preliminary study has examined protein expression in EGC in human intestinal biopsy samples from IBS patients, without observing any changes. $34 \mathrm{~A}$ recent study performed in an animal model of IBS (maternal separation fol- lowed by acute stress) reported structural changes in EGC, such as elongation and/or swelling of terminal processes.15 Alterations of glial markers have been reported in other diseases such as inflammatory bowel diseases, where a dual expression phenotype was identified, namely an increased expression of $S 100 \beta$ and GFAP in inflamed areas of ulcerative colitis tissues compared to controls, whereas a reduced

S100 $\beta$ and GFAP expression was observed in non-inflamed areas of Crohn's disease tissues. Surprisingly, in this latter setting, there were no changes in S100 $\beta$ and GFAP expression in inflamed areas vs controls.16 This finding is in line with our previous data showing no changes in S100 $\beta$ or GFAP expression in EGC isolated from Crohn's disease tissues, although arachidonic acid metabolites were reduced in vitro.35 Taken together, these results suggest that EGC could display an altered functional response in IBS regardless of an apparently normal expression of $\mathrm{S} 100 \beta$ and GFAP.

Using a validated model of adoptive transfer of disease, we showed that 'long-term' (ie, 48 hours) incubation of EGC with IBS supernatants did not alter the expression of $S 100 \beta$ and GFAP in EGC. We studied the impact of 'long-term' exposure of EGC to supernatants as this approach could allow remodeling of EGC phenotype or functions, which cannot be observed during acute exposure of EGC to supernatants. This is further supported by recent findings demonstrating that chronic exposure of enteric neurons to IBS supernatants reduces rather than increases neuronal excitability. 36 In this context, our results showed that long- term incubation of EGC with IBS-C supernatants, but not IBS-D or IBS- M, reduced EGC number in vitro. This effect was not associated with an increased cell death suggesting that IBS supernatants can affect the EGC cell cycle. Whether this property is specific to IBS-C 
subgroup should be confirmed on a larger population of IBS patients. However, it is intriguing to observe that (i) animal models leading to a reduction in EGC number or inhibition of their functions result in reduced gut motility and (ii) motility disorders, such as severe slow transit constipation, are characterized by a reduced EGC number. Therefore, it is tempting to speculate that approaches aimed to stimulate EGC proliferation may turn to be useful in motility dysfunctions. The mediator(s) involved in these effects remain(s) to be determined, as histamine did not induce any changes in EGC number. Previous studies have shown that cyto- kines such as IL1ß, IL-4, IFN- $\gamma$, or lipopolysaccharide can modulate EGC proliferation in vitro but their involvement in the present context need to be tested in future studies. A limitation of our study is that the ef- fects of exposure to IBS supernatants were only explored in vitro and in rat EGC isolated from the myenteric plexus. Therefore, whether similar changes occur in vivo and can be extended to other EGC types, such as of submucosal origin, remains to be determined in future studies.

Another major finding of our study was that IBS-D and IBS-M supernatants significantly reduced $\mathrm{Ca} 2+$ response to ATP in EGC as compared to HC. Recent studies have shown the importance of $\mathrm{Ca} 2+$ response to ATP in enteric neuroglial communication and ultimately in control of gastrointestinal functions controlled by the ENS.37 Indeed, ATP has been shown to activate $\mathrm{P} 2 \mathrm{Y} 4$ receptors leading to an increase of intracellular $\mathrm{Ca} 2+$ concentration in $\mathrm{EGC}$, which propagates to the functional EGC syncytium via connexin-43, and ultimately impacting on $\mathrm{GI}$ motility. McClain et al. also reported reduced expression of connexin-43 associated with reduced glial $\mathrm{Ca} 2+$ response and altered motility in aging mice.24 Interestingly, $\mathrm{Ca} 2+$ response to ATP in EGC treated with IBS supernatants was negatively correlated with pain further reinforcing the hypothesis of a functional link between EGC and symptoms. In order to determine mechanisms potentially involved in altered Ca2+ response to ATP by IBS supernatants, we first aimed at determining whether changes in EGC expression of ATP receptors (P2Y) could be involved. However, incubation of EGC with supernatants did not modify P2Y4 mRNA expression. Connexin-43 mRNA expression increased in EGC incubated with IBS supernatants, which was specific for IBS-C and IBS-D but not IBS-M. Whether this increased expression of connexin-43 contributes to maintain a normal Ca2+ response to ATP in EGC incubated with IBS-C supernatants or is involved in supernatant-induced reduction in EGC number remains to be determined. Alternatively, the reduced $\mathrm{Ca} 2+$ response to ATP could also be a consequence of a general change in the cellular $\mathrm{Ca} 2+$ machinery. Altogether these results suggest that altered ATP-induced Ca2+ signaling in EGC could contribute to GI dysfunctions observed in IBS.

An important finding of our study was the identification of histamine as a key soluble factor of the mucosal microenvironment involved in EGC dysfunctions induced by IBS supernatants. Previous studies have reported an increased concentration of histamine in IBS supernatants.38 Interestingly, increased mRNA expression of H1R and H2R was also reported in IBS digestive mucosa. 39 Histamine is mainly secreted by mast cells 40 but food and enteric bacteria are also alternative sources of histamine. Interestingly, a recent study has shown that $58 \%$ of IBS patients reported GI symptoms from histamine-releasing food components.41 The involvement of histamine in the pathophysiology of IBS has been identified in earlier studies3,42-44 and emerges as a promising therapeutic target for treating IBS-related symptoms.45-48 In the ENS, histamine has been shown to contribute to increase neuronal activity induced by IBS supernatants.38,49,50 Histamine affects enteric neurons via H1R, $\mathrm{H} 2 \mathrm{R}$, and $\mathrm{H} 3 \mathrm{R}$, as demonstrated by the use of selective an- tagonists (respectively pyrilamine, ranitidine, and clobenpropit).38 To date, the functional impact of histamine 
upon EGC remained largely unknown. Kimball et al. reported that acute application of histamine to EGC increased intracellular Ca2+ concentration but the receptors in- volved remained unknown. 51 In contrast in preliminary tests, we were unable to detect a Ca2+ response induced by acute application of his- tamine in EGC. This could also explain the absence of $\mathrm{Ca} 2+$ response to acute exposure of EGC to IBS supernatants. However, our study adds to the role of histamine onto EGC functions by demonstrating that (i) EGC expresses $\mathrm{H} 1 \mathrm{R}$ and (ii) EGC long-term exposure to histamine reduces Ca2+ response to ATP via H1R. Although the mechanisms responsible for histamine effects remain to be determined, they probably do not result from histamine-induced reduction in the expression of puriner-gic receptors. Alternatively, histamine could also reduce ATP-induced extracellular $\mathrm{Ca} 2+$ entry or $\mathrm{Ca} 2+$ release by intracellular stores such as endoplasmic reticulum. Interestingly, while the H1R antagonist pyril- amine completely abolished histamine effects upon Ca2+ response to ATP, it only partially inhibited the effect of IBS supernatants. This latter result suggests that, in addition to histamine, other soluble factors may contribute to the effects of supernatant upon Ca2+ responses to ATP.52

Altogether, our study identified EGC as a novel cellular component that can contribute to IBS pathophysiology and symptom develop- ment. Finally, distinct EGC-related mechanisms may influence the IBS phenotype and thereby symptom pattern.

\section{ACKNOWLEDGMENTS}

The authors thank the Research Nurses at the Centre d'Investigation Clinique (CIC), as well as the endoscopic staff (physicians and nurses) who participated to the study at the University Hospital of Nantes.

\section{CONFLICT OF INTEREST}

No competing interests exist.

\section{AUTHOR CONTRIBUTIONS}

NLL, LQ, SH, and PA contributed to study concept and design; TD was responsible for acquisition of data; NLL, LQ, SH, PA, TD were respon- sible for analysis and interpretation of data; NLL, CB, SBV, EC, and MN contributed to drafting of the manuscript; $L Q, C B, S B V, E C$, $M N, R D G$, and $G B$ were responsible for critical revision of the manuscript for important intellectual content; $\mathrm{CB}, \mathrm{MN}$, and JBH was responsible for statistical analysis; $\mathrm{MN}$ obtained funding; MRD and PN provided administrative, technical, or material support; and EC and $\mathrm{MN}$ were responsible for study supervision. All authors approved of the final version of the manuscript.

\section{REFERENCES}

1. Lovell RM, Ford AC. Global prevalence of and risk factors for irri- table bowel syndrome: a meta-analysis. Clin Gastroenterol Hepatol. 2012;10:e4.

2. Plavšić $\mathrm{I}$, Hauser $\mathrm{G}$, Tkalčić M, et al. Diagnosis of irritable bowel syndrome: role of potential biomarkers. Gastroenterol Res Pract. 2015;2015:490183.

3. Barbara G, Stanghellini V, De Giorgio R, et al. Activated mast cells in proximity to colonic nerves correlate with abdominal pain in irritable bowel syndrome. Gastroenterology. 2004;126:693-702.

4. Macsharry J, O'Mahony L, Fanning A, et al. Mucosal cytokine imbalance in irritable bowel syndrome. Scand J Gastroenterol. 2004;43:1467-1476.

5. Piche T, Barbara G, Aubert $P$, et al. Impaired intestinal barrier integrity in the colon of patients with irritable bowel syndrome: involvement of soluble mediators. Gut. 2009;58:196-201. 
6. Park JH, Rhee PL, Kim G, et al. Enteroendocrine cell counts cor- relate with visceral hypersensitivity in patients with diarrhoea- predominant irritable bowel syndrome. Neurogastroenterol Motil Off J Eur Gastrointest Motil Soc. 2006;18:539-546.

7. Dothel $\mathrm{G}$, Barbaro $\mathrm{R}$, Boudin $\mathrm{H}$, et al. Nerve fiber outgrowth is in- creased in the intestinal mucosa of patients with irritable bowel syn- drome. Gastroenterology. 2015;148:1002-1011.

8. Wang LH, Fang XC, Pan GZ. Bacillary dysentery as a causative factor of irritable bowel syndrome and its pathogenesis. Gut. 2004;53:1096-1101.

9. Akbar A, Yiangou Y, Facer P, et al. Increased capsaicin receptor TRPV1-expressing sensory fibres in irritable bowel syndrome and their correlation with abdominal pain. Gut. 2008;57:923-929.

10. Buhner $S$, Li Q, Berger T, et al. Submucous rather than myenteric neurons are activated by mucosal biopsy supernatants from irri- table bowel syndrome patients. Neurogastroenterol Motil Off J Eur Gastrointest Motil Soc. 2012;24:1134-e572.

11. Buhner S, Schemann M. Mast cell-nerve axis with a focus on the human gut. Biochim Biophys Acta. 2012;1822:85-92.

12. Zeller $F$, Von Weyhern CW, et al. Quantitative assessment of glial cells in the human and guinea pig enteric nervous system with an anti- Sox8/9/10 antibody. J Comp Neurol. 2008;509:356-371.

13. Grubišić V, Gulbransen BD. Enteric glia: the most alimentary of all glia. J Physiol. 2017;595:557-570.

14. Neunlist M, Rolli-Derkinderen M, Latorre R, et al. Enteric glial cells: recent developments and future directions. Gastroenterology. 2014;147:1230-1237.

15. Fujikawa Y, Tominaga K, Tanaka F, et al. Enteric glial cells are associ- ated with stress-induced colonic hypercontraction in maternally sep- arated rats. Neurogastroenterol Motil Off J Eur Gastrointest Motil Soc. 2015;27:10101023.

16. Cornet A, Savidge TC, Cabarrocas J, et al. Enterocolitis induced by autoimmune targeting of enteric glial cells: a possible mechanism in Crohn's disease? Proc Natl Acad Sci USA. 2001;98:13306-13311.

17. Aubé AC, Cabarrocas J, Bauer J, et al. Changes in enteric neurone phenotype and intestinal functions in a transgenic mouse model of enteric glia disruption. Gut. 2006;55:630-637.

18. Fletcher EL, Clark MJ, Furness JB. Neuronal and glial localization of GABA transporter immunoreactivity in the myenteric plexus. Cell Tissue Res. 2002;308:339-346.

19. Zhou X, Galligan JJ. GABA(A) receptors on calbindin-immunoreactive myenteric neurons of guinea pig intestine. J Auton Nerv Syst. 2000;78:122-135.

20. Nagahama M, Semba R, Tsuzuki M, et al. L-arginine immunoreac- tive enteric glial cells in the enteric nervous system of rat ileum. Biol Signals Recept. 2001;10:336-340.

21. Gomes P, Chevalier J, Boesmans W, et al. ATP-dependent paracrine communication between enteric neurons and glia in a primary cell cul- ture derived from embryonic mice. Neurogastroenterol Motil Off J Eur Gastrointest Motil Soc. 2009;21:870-e62.

22. van Nassauw L, Costagliola A, Op Van, den Bosch J, et al. Region- specific distribution of the P2Y4 receptor in enteric glial cells and interstitial cells of Cajal within the guinea-pig gastrointestinal tract. Auton Neurosci Basic Clin. 2006;126127:299-306.

23. Hanani M, Zamir O, Baluk P. Glial cells in the guinea pig myenteric plexus are dye coupled. Brain Res. 1989;497:245249.

24. McClain JL, Grubišić V, Fried D, et al. $\mathrm{Ca}^{2+}$ responses in enteric glia are mediated by connexin-43 hemichannels and modulate colonic transit in mice. Gastroenterology. 2014;146:497-507.

25. Nasser $Y$, Fernandez E, Keenan CM, et al. Role of enteric glia in in- testinal physiology: effects of the gliotoxin fluorocitrate on motor and secretory function. Am J Physiol Gastrointest Liver Physiol. 2006;291:912-927.

26. Bassotti $G$, Villanacci $V$, Maurer $C A$, et al. The role of glial cells and apoptosis of enteric neurones in the neuropathology of intractable slow transit constipation. Gut. 2006;55:41-46.

27. Iantorno G, Bassotti G, Kogan Z, et al. The enteric nervous system in chagasic and idiopathic megacolon. Am J Surg Pathol. 2007;31:460- 468.

28. Bohórquez DV, Samsa LA, Roholt A, et al. An enteroendocrine cell- enteric glia connection revealed by 3D electron microscopy. PLOS ONE. 2014;9:e89881.

29. Neunlist $M$, Van Landeghem L, Mahé MM, et al. The digestive neuronal-glial-epithelial unit: a new actor in gut health and disease. Nat Rev Gastroenterol Hepatol. 2013;10:90-100.

30. Drossman DA. The functional gastrointestinal disorders and the Rome III process. Gastroenterology. 2006;130:13771390.

31. Lebouvier $\mathrm{T}$, Coron $\mathrm{E}$, Chaumette $\mathrm{T}$, et al. Routine colonic biop- sies as a new tool to study the enteric nervous system in living patients. Neurogastroenterol Motil Off J Eur Gastrointest Motil Soc. 2010;22:e11-e14.

32. Chevalier J, Derkinderen P, Gomes P, et al. Activity-dependent regula- tion of tyrosine hydroxylase expression in the enteric nervous system. J Physiol. 2008;586:1963-1975.

33. Van Landeghem L, Chevalier J, Mahé MM, et al. Enteric glia pro- mote intestinal mucosal healing via activation of focal adhesion ki- nase and release of proEGF. Am J Physiol Gastrointest Liver Physiol. 2011;300:G976-G987.

34. Andrews CN, Shaffer E, Ho W, Nasser Y, et al. Enteric glia and en- teroendocrine cells in irritable bowel syndrome: controlled Pilot Study (Abstract). Gastroenterology. 2005;128:A-270.

35. Pochard C, Coquenlorge S, Jaulin J, et al. Defects in 15 -HETE pro- duction and control of epithelial permeability by human enteric glial cells from patients with crohn's disease. Gastroenterology. 2016;150:168-180.

36. Ostertag D, Buhner $S$, Michel K, et al. Reduced responses of submu- cous neurons from irritable bowel syndrome patients to a cocktail containing histamine, serotonin, TNF $\alpha$, andTryptase (IBS-Cocktail). Front Neurosci. 2015;16:465. 
37. Gulbransen BD, Sharkey KA. Purinergic neuron-to-glia signaling in the enteric nervous system. Gastroenterology. 2009;136:1349-1358

38. Buhner S, Li Q, Vignali S, et al. Activation of human enteric neurons by supernatants of colonic biopsy specimens from patients with irritable bowel syndrome. Gastroenterology. 2009;137:1425-1434.

39. Sander LE, Lorentz A, Sellge G, et al. Selective expression of histamine receptors $H 1 R, H 2 R$, and $H 4 R$, but not $H 3 R$, in the human intestinal tract. Gut. 2006;55:498-504.

40. Jutel $\mathrm{M}, \mathrm{Akdis} \mathrm{M}, \mathrm{Akdis} C \mathrm{~A}$. Histamine, histamine receptors and their role in immune pathology. Clin Exp Allergy $\mathrm{JBr}$ Soc Allergy Clin Immunol. 2009;39:1786-1800.

41. Böhn L, Störsrud S, Törnblom H, et al. Self-reported food-related gastrointestinal symptoms in IBS are common and associated with more severe symptoms and reduced quality of life. Am J Gastroenterol. 2013;108:634-641.

42. Thomas $\mathrm{CM}$, Hong T, van Pijkeren JP, et al. Histamine derived from probiotic Lactobacillus reuteri suppresses TNF via modulation of PKA and ERK signaling. PLoS ONE. 2012;7:e31951.

43. Masaki T, Yoshimatsu $\mathrm{H}$. The hypothalamic $\mathrm{H} 1$ receptor: a novel therapeutic target for disrupting diurnal feeding rhythm and obesity. Trends Pharmacol Sci. 2006;27:279-284.

44. Hattori $\mathrm{T}$, Watanabe $\mathrm{S}$, Kano $\mathrm{M}$, et al. Differential responding of autonomic function to histamine $\mathrm{H}$ antagonism in irritable bowel syndrome. Neurogastroenterol Motil Off J Eur Gastrointest Motil Soc. 2010;22:1284-1291.

45. Klooker TK, Braak B, Koopman KE, et al. The mast cell stabiliser ketotifen decreases visceral hypersensitivity and improves intes- tinal symptoms in patients with irritable bowel syndrome. Gut. 2010;59:1213-1221.

46. Tack JF, Miner PB, Fischer L, et al. Randomised clinical trial: the safety and efficacy of AST-120 in non-constipating irritable bowel syndrome - a double-blind, placebo-controlled study. Aliment Pharmacol Ther. 2011;34:868-877.

47. Stanisor OI, Van Diest SA, Yu Z, et al. Stress-induced visceral hy- persensitivity in maternally separated rats can be reversed by pe- ripherally restricted histamine-1-receptor antagonists. PLOS ONE. 2013;8:e66884

48. 48. Wouters MM, Balemans D, Van Wanrooy S, et al. Histamine recep- tor H1-mediated sensitization of TRPV1 mediates visceral hyper- sensitivity and symptoms in patients with irritable bowel syndrome. Gastroenterology. 2016;150(875-887):e9.

49. 49. Barbara G, Wang B, Stanghellini V, et al. Mast cell-dependent exci- tation of visceral-nociceptive sensory neurons in irritable bowel syn- drome. Gastroenterology. 2007;132:26-37.

50. 50. Breunig $E$, Michel $K$, Zeller $F$, et al. Histamine excites neurones in the human submucous plexus through activation of $\mathrm{H} 1, \mathrm{H} 2, \mathrm{H} 3$ and $\mathrm{H} 4$ receptors. J Physiol. 2007;583:731-774.

51. 51. Kimball BC, Mulholland MW. Enteric glia exhibit P2U receptors that increase cytosolic calcium by a phospholipase C-dependent mecha- nism. J Neurochem. 1996;66:604.

52. 52. Ostertag D, Buhner $S$, Michel K, et al. Reduced responses of submu- cous neurons from irritable bowel syndrome patients to a cocktail containing histamine, serotonin, TNF $\alpha$, and Tryptase (IBS-Cocktail). Neurosci. 2015;9:465. 